Personalie

\section{Peter Braun wird CEO der CAMLOG Gruppe}

Herr Peter Rudolf Braun ist per 1. Juni 2014 neu als Chief Executive Officer bei der CAMLOG Biotechnologies AG in Basel, Schweiz, eingetreten. Er übernimmt die operative Führung von Interims-CEO Dr. René Willi, dessen Kompetenz als Mitglied und Delegierter des Verwaltungsrats der CAMLOGGruppe weiterhin zur Verfügung steht. Jürg Eichenberger, erfolgreicher Unternehmer mit langjährigen Erfahrungen in Unternehmensgründungen und -führung bekleidet seit 2003 das Amt des Präsidenten des Verwaltungsrats der CAMLOG Holding. Unter seiner Leitung ist der Verwaltungsrat zuständig für die strategi-

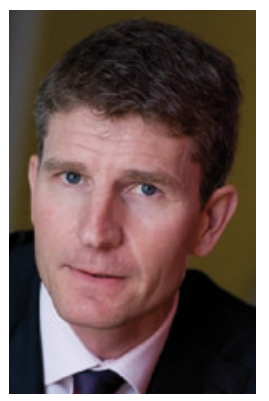

sche Ausrichtung der international tätigen CAMLOG-Gruppe. Herr Braun wird die Erfolgsgeschichte von CAMLOG mit seinen ausgezeichneten strategischen, operativen und organisatorischen Fähigkeiten weiter schreiben. Seine breite Erfahrung in der HealthcareIndustrie will er nutzen, um die Position von CAMLOG im Markt der dentalen Implantologie weiter zu stärken. Eines seiner Hauptziele wird die weitere Internationalisierung der Marke CAMLOG sein.

Nach einer Pressemitteilung der

CAMLOG BiotechnologiesAG, CH-Basel

Internet: www.camlog.com

\section{Die korrekte Abrechnung - ein Erfolgspotenzial}

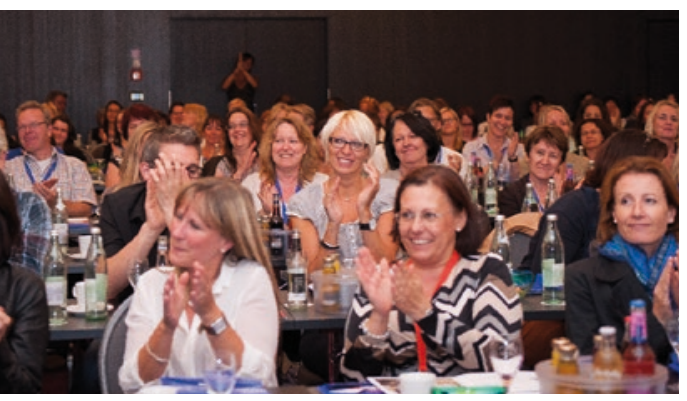

Verborgene Potenziale entdecken und erfolgreich heben. Darum ging es während des 2. Dr. Güldener-Kongress für Abrechnung und Praxismanagement am 27. und 28. Juni im Stuttgarter Mövenpick Hotel. „Als Abrechnungsdienstleister sehen wir, dass diese Bereiche neben der Behandlungskompetenz einer der herausragenden Faktoren für eine erfolgreiche Praxis sind. Deshalb wollen wir das dafür notwendige Know-how mit Fortbildungen und Kongressen wie diesem weiter fördern“, so Dr. Peter Raab, Moderator der Veranstaltung. Mit Erfolg, denn mit den rund 230 Teilnehmern war der Kongress ausgebucht - darunter Abrechnungsspezialistinnen, Praxismanagerinnen und Praxisinhaber. Die Themen waren weit gefasst: Von der Honorargestaltung für GKVund PKV-Patienten über Praxisorganisation, Patientenkommunikation und Mar- keting bis hin zu rechtlichen Aspekten. Die exzellenten Referenten vermittelten den Teilnehmern ein ganzheitliches Verständnis für das Managen einer Praxis. Das Fazit des Kongresses fällt eindeutig aus: Die Teilnehmer haben von den Vorträgen und teilweise regen Diskussionen mit den Experten auf der Bühne sehr profitiert. Die Referenten haben es verstanden, das große Potenzial des Berufsbilds Praxismanager für den Praxiserfolg informativ und auch unterhaltsam darzustellen. So stand am Ende für die Teilnehmerinnen und Teilnehmer fest: Sie sind im nächsten Jahr wieder dabei.

Nach einer Pressemitteilung der

Deutsches Zahnärztliches Rechenzentrum

GmbH, Stuttgart

Internet: www.dzr.de
Ausschreibung

\section{Forschungspreis}

\section{Vollkeramik 2015}

Seit 15 Jahren verleiht die Arbeitsgemeinschaft für Keramik in der Zahnheilkunde (AG Keramik) alljährlich den „Forschungspreis Vollkeramik“. Nun tritt die AG Keramik mit der Ausschreibung erneut an die Fachwelt heran. Der Forschungspreis soll Zahnärzte sowie Wissenschaftler und besonders interdisziplinäre Arbeitsgruppen motivieren, Arbeiten zum Werkstoff Keramik und zu vollkeramischen Restaurationen einzureichen. Im Rahmen des Themas werden klinische Untersuchungen angenommen, die auch die zahntechnische Ausführung im Labor umfassen. Deshalb können auch die ausführenden Zahntechniker als Mitglieder von Arbeitsgruppen teilnehmen. Materialtechnische Untersuchungen mit Vollkeramiken sind ebenfalls im Fokus der Ausschreibung. Ferner werden klinische Arbeiten geschätzt, die sich mit der computergestützten Fertigung (CAD/CAM) und Eingliederung von vollkeramischen Kronen, Brücken und Implantat-Suprakonstruktionen befassen.

Bis zum 28. Februar 2015 einreichen In der neuen Ausschreibung zum Forschungspreis 2015 werden noch nicht eingereichte wissenschaftliche Arbeiten und klinische Untersuchungen angenommen. Der Forschungspreis ist mit 5000,-€ dotiert. Einsendeschluss ist der 28. Februar 2015 (Poststempel). Die Arbeit wird vom unabhängigen wissenschaftlichen Beirat der AG Keramik bewertet. Weitere Informationen erhalten Sie per Telefon (0721) 9452929, Fax (0721) 9452930, per Email: info@ag-keramik.de oder im Web www.ag-keramik.de

Nach einer Pressemitteilung der AG Keramik, Ettlingen
ZWR - Das deutsche Zahnärzteblatt

\section{Neue Bezugspreise ab 1. Januar 2015}

Die Kosten für unsere Zeitschriften sind auch im vergangenen Jahr gestiegen. Wir bitten daher um Verständnis, wenn wir uns gezwungen sehen, den Bezugspreis für die ZWR - Das deutsche Zahnärzteblatt ab 1. Januar 2015 neu festzusetzen. Die Preise sind wie folgt: Institutioneller Jahresbezugspreis (includiert Online-Zugriff für 1 Standort) $379,-€$ und persönlicher Jahresbezugspreis $247,-€$ (Vorzugspreis für Ärzte in der Weiterbildung und Studenten 111,- €), jeweils zzgl. Versandkosten (Inland 45,90€, Europa 58,80€, Übersee 96,80€); Einzelheftpreis 34,-€, zzgl. Versandkosten ab Verlagsort. 